\title{
Remote support to victims of violence against women and domestic violence during the COVID-19 pandemic
}

\author{
Sónia Maria Martins Caridade, Rosa Saavedra, Rita Ribeiro, Ana Cristina Oliveira, \\ Manuela Santos, Iris Sofia Almeida and Cristina Soeiro
}

Received 30 April 2021 Revised 25 June 2021 5 July 2021

Accepted 14 July 2021

(C) Sónia Maria Martins Caridade, Rosa Saavedra, Rita Ribeiro, Ana Cristina Oliveira,

Manuela Santos, Iris Sofia

Almeida and Cristina Soeiro.

Published by Emerald

Publishing Limited. This article

is published under the Creative Commons Attribution (CC BY

4.0) licence. Anyone may

reproduce, distribute, translate and create derivative works of this article (for both commercial \& non-commercial purposes), subject to full attribution to the original publication and authors. The full terms of this licence may be seen at http:// creativecommons.org/licences/ by/4.0/legalcode

Conflicts of interest: The authors declared no potential conflicts of interest with respect to the research, authorship and/or publication of this article.

Funding: This study was supported by the Foundation for Science and Technology (FCT) and Commission for Citizenship and Gender Equality (CIG) through the Portuguese State Budget (GenderResearch4Covid9598419).

\begin{abstract}
Purpose - This paper aims to characterize the type of support provided to victims of violence against women and domestic violence (VAWDV) during the first lockdown, assessing the training of professionals to use remote support (RS).

Design/methodology/approach - This cross-sectional study involves a sample of 196 support professionals, mainly women (91.8\%) and who integrate the Portuguese National Support Network for victims of domestic violence (NSNVDV) (Mean age $=36.49$; $S D=10.52$ ).

Findings - Telephone emerges as the main RS communication media used in the lockdown (43.9\%) and the emergency state periods (57.1\%). Participants reported to have never used any social applications (41.8\% vs $41.8 \%$ ) or videoconference (46.4\% vs 58.2\%), in both periods assessed, i.e. lockdown and emergency state, respectively, and $82.7 \%$ assumed to have no training with RS to assist VAWDV victims. However, support professionals recognized several advantages in using RS such as dealing with isolation, reducing inhibition, fear and shame and in promoting the victims' empowerment.

Research limitations/implications - Given the exploratory nature of this study, only descriptive analyzes were conducted.

Originality/value - During the COVID-19 pandemic, little is known about effective RS given by professionals to victims of VAWDV in the Portuguese context. The paper aims to add knowledge to the studied field.
\end{abstract}

Keywords Victims, Lockdown, COVID-19 pandemic, Remote support (RS), Support professionals, Violence against women and domestic violence (VAWDV)

Paper type Research paper

\section{Introduction}

Violence against women and domestic violence (VAWDV) has been widely described as a worldwide problem and an important issue involving human rights and public health. More specifically and according to the United Nations (1993), violence against women may involve any "act of gender-based violence that results in or is likely to result in, physical, sexual or mental harm or suffering to women, including threats of such acts, coercion or arbitrary deprivation of liberty, whether occurring in public or in private life." Although domestic violence (DV) is often associated with the occurrence of violence in intimate relationships (Murray et al., 2015), it refers not only to violence between intimate partners and but also includes children and the abuse of elderly people or any other members of the family in Portugal (Law 19/2013).

Women have been identified as the main victims of DV, with worldwide estimates indicating that $30 \%$ of women have already experienced some type of physical and/or sexual violence (Trabold et al., 2018). The Center for Disease Control and Prevention (2016) documents that 
one in five women and one in seven men who have experienced some kind of rape, physical abuse and harassment by an intimate partner have also experienced some type of intimate violence between 11 and 17 years of age. WHO (2013) also reported that one in three women has already experienced some type of physical and/or sexual violence by their intimate partner. A survey on violence against women conducted by the European Union with 42,000 women from 28 European states found interesting results, namely, that 1 in 3 women suffered at least one event of physical and or sexual violence, as the age of 15 , $2 \%$ of women experience physical or sexual violence by a partner and $11 \%$ of women have experienced some form of sexual violence (FRA, 2014). In Portugal, similarly to what was registered in other European countries (FRA, 2014), high rates of intimate violence have been identified, both among adults (Neves and Nogueira, 2010) as among adolescents (Faias et al., 2016; Neves et al., 2016). The official Portuguese data shows that in 2020, DV reached the highest levels since 2010 , constituting the most reported crime against people, with 23,439 cases. Women represented $75 \%$ of the total victims, with $14.3 \%$ under 16 years old and $11.6 \%$ between 16 and 24 years old and $74 \%$ being 25 years old or more. Men represented $81.4 \%$ of all aggressors (SSI - Sistema de Segurança Interna, 2021).

\section{The impact of the coronavirus pandemic (COVID-19)}

The outbreak of the coronavirus pandemic (COVID-19) has been identified and had serious negative consequences for victims of intimate partner violence (IPV) (Boserup et al., 2020; Bradbury-Jones and Isham, 2020; EIGE, 2021; Usher et al., 2020), as well as enhancing the prevalence and severity rates of IPV, namely, due to the restrictions imposed, e.g. isolation, social distance and its potential impact on the request for help by victims. A recent Portuguese study (Paulino et al., 2021) with a sample of 10,529 participants, with $83.5 \%$ women, found a moderate or severe psychological impact of the outbreak in $49.2 \%$ of the participants. Depression (11.7\%), anxiety (16.9\%) and stress were rated as moderate to severe problems. Effectively, women and children have been identified as constituting one of the groups most affected by the implications of the COVID-19 pandemic, similarly to what was seen in other disasters, either natural or not (Yenilmez, 2020). Another study by Walklate et al. (2021) analyzed and debated the potential impact of COVID-19 and the associated public health response in increasing the violence experienced by women and children in their homes and greater complexity of the experienced impacts, which require responses that imply a broader structural and organizational change. Several factors have been associated with an increased risk of VAWDV during the period of the first Portuguese lockdown (March to May 2020). Examples are the fact that the aggressor and victim are forced to live long periods of time together; the victim's social isolation inevitably leads to less informal control of the perpetrator by the community, interfering with the victim's requests for help; the increase of social stressors associated with DV, e.g. economic problems or job insecurity, which can more easily enhance the aggressor's lack of control by using violence; or boosting alcohol consumption by the DV aggressor (Boxall et al., 2020).

In addition, the restrictions imposed on the COVID-19 lockdown also impacted the use of community resources, placing additional pressure on some of the most vulnerable, including victims (and potential perpetrators) of domestic abuse (Anka et al., 2020), as well in the response given by institutions and professionals working with the victims. In fact, professionals are faced with challenging tasks, such as predicting the potential consequences of the instability that was felt during the pandemic. They were forced to find new models to support victims, fulfilling the requirements arising from the pandemic (Anka et al., 2020). Considering the restrictions associated with formal and informal help, remote working using digital technological platforms such as Zoom, Skype and telephone, emerged as necessary alternatives in the support of victims in situations of crisis (Anka et al., 2020; Bocioaga, 2020; Safe Lives, 2020). It was also found that face-to-face (f2f) assistance was restricted due to the nature of pandemic circumstances, being necessary to 
reconfigure the service model for remote and/or online interventions. A study conducted by Safe Lives (2020) comprising service providers in cases of DV $(n=119)$ illustrates a significant reduction in the provision of services (76\%) during COVID-19, with $88 \%$ canceling f2f assessments and $86 \%$ of professionals working from home, thus corroborating the difficulties brought by the pandemic.

\section{Remote support to violence against women and domestic violence victims}

The increased use of information and communication technologies (ICT) by the population in general, combined with the emergence of a pandemic situation generated by COVID-19, emphasized the need to consider the use of digital solutions to support victims of VAWDV.

Nevertheless, the use of ICT in response to VAWDV already existed before the COVID-19 pandemic situation and digital-based programs to primarily address IPV has emerged in the past years (Anderson et al., 2019; Constantino et al., 2014; Ford-Gilboe et al., 2017; Glass et al., 2015; Hegarty et al., 2019; Sabri et al., 2019). In the case of aggressors, there are already technological devices to control them (e.g. use of tags/tracking) and some technological applications for screening and referencing cases of DV have also emerged in the past years (Murray et al., 2015). As an example, a computer application in the Portuguese context, APP-VD, was recently launched by the Commission for Citizenship and Gender Equality (2020) to promote the request for help in cases of DV. Indeed, it has been documented that ICT may be an important tool in supporting victims of VAWDV and other violence, enabling not only the rapid dissemination of information on this type of violence, essential resources and support services for victims but also allowing the creation of online support groups to reduce the feeling of isolation, provide victims with security devices and safety plans and, additionally, the empowerment of victims (Al-Alosi, 2020). Other benefits have been associated with digital solutions in support of the victim. For example, it has been demonstrated that interventions in online environments make it possible to better deal with social risk and inhibition, reducing them and promoting the sharing of unwanted thoughts and feelings (Constantino et al., 2014), thus allowing the victims to feel free to ask for help. In addition, and on the side of the professionals who provide support to the victim of VAWDV, ICT-based solutions allow managing the difficulties of participating in prevention and f2f training initiatives for support professionals, such as worker's safety, travel restrictions and limited funding (Runyan et al., 2005).

Notwithstanding the many opportunities that digital solutions (e.g. webinars; mobile phone technology including email, live video interaction or mobile app; media/communication platforms such as Facebook, Instagram and WhatsApp) can have in supporting victims of VAWDV, they also have several challenges that require careful management. Problems such as user security, user load, data privacy and confidentiality have been identified as important challenges and priorities to be considered in remote support (RS) (Murray et al., 2015). Despite the widespread dissemination of digital practices in society, there are groups and victims who experience significant barriers of different order in accessing and handling digital tools. Examples that may be used to illustrate this are as follows: older people show less skill in handling ICT; residents in rural areas may not have a suitable internet signal; the precarious economic condition makes it difficult to access digital devices or yet; reduced literacy that can interfere with the will and/or skills of people and victims, in particular, to use the technology to seek help or select the most appropriate information for the support they need. The availability, training and practice of support professionals for the adoption of technology and incorporate it in the provision of services or even the financing options that should be available for this purpose are other challenges to be considered (Al-Alosi, 2020). The safety of IPV victims is another important challenge, as offenders can remotely monitor their devices, such as computers, phones and home security systems, locating victims, and thus putting their physical security at risk (Finn and Atkinson, 2009). 
Finding strategies or control mechanisms for the most diverse challenges and risks inherent to the use of ICT, have the potential to be key tools to combat VAWDV, to take advantage of the enormous potential of RS, specifically in a pandemic situation such as the one being currently experienced (Caridade and Dinis, 2020). On the other hand, and despite the increase in digital solutions, they should not replace f2f support, but rather constitute an important alternative and complement. There is, therefore, an urgent need to develop financing policies that can increase the type of response given by the services to more solidly implement RS (Chayn and SafeLives, 2017).

\section{The present study}

Studies focusing on the use of ICT by professionals supporting victims of VAWDV in the Portuguese context are unknown, so this study appears to be innovative from this point of view, also constituting the first one to analyze the type of support provided to victims of VAWDV during the COVID-19 pandemic situation. It has been argued that the provision of RS poses several challenges to service providers, raising concerns about the confidentiality for victims' testimony, employees, the access to necessary equipment, ICT capabilities and experience or training by support professionals, as well as it proves to be a much more demanding task for professionals who face the need to impose work-life limits and to manage increasing levels of stress (EIGE, 2021). In addition, the situation of "living with COVID-19" must be considered (Anka et al., 2020, p. 424), from the point of view of professionals supporting victims of VAWDV, for example, to identify the level of preparation they have and training needs. Accordingly, the present study has the general objective of characterizing the RS given by the support professionals of the Portuguese National Support Network for victims of domestic violence (NSNVDV), with a particular focus on the period of lockdown and emergency state imposed by the pandemic of COVID-19 in Portugal. Specifically, it is intended to as follows:

- Identify the type of support most used by professionals assisting the victims;

- Characterize the type of support most provided in the lockdown period;

- Identify the RS modalities used during the state of emergency;

- Characterize the training of professional support for victims in RS, as well as training needs in this area; and

- Understand the institutional policies supporting the implementation of RS.

The following hypotheses are investigated:

H1. Telephone as RS media is mostly used by support professionals to assist victims of VAWDV, both in general as during the pandemic period.

H2. Support professionals have less training and preparation in the use of RS to provide support to victims of VAWDV.

H3. Institutional policies supporting the use of RS to assist victims of VAWDV are restricted.

\section{Method \\ Sample}

This study included a convenience sample of 196 professionals supporting the victims of VAWDV, mainly women $(91.8 \%$ ) who are part of the NSNVDV, with a mean age of 36.49 $(S D=10.52)$. As expected, most participants $(99 \%)$ have higher education, with $56.6 \%$ of the participants having a degree in psychology, $18.4 \%$ in law and $15.8 \%$ in social work. In total, $86.2 \%$ of the participants reported they had training as victim support technicians with 
the majority (74\%) carrying out functions in the context of a victim support institution and with a mean of $4.88(S D=5.65)$ years of professional experience.

\section{Procedure}

The questionnaire protocol was initially made available through an online survey on the Google Docs online platform. For the dissemination of the uniform resource locator with the questionnaire, collaboration with the Commission for Citizenship and Gender Equality was requested, so that it was possible to reach the largest possible number of professionals who support victims of VAWDV within the scope of NSNVDV. Before asking the participants to complete the questionnaire, a brief description of the study was presented, clarifying the objectives, the inclusion criterion (i.e. support professionals within the NSNVDV, in Portugal), the confidentiality and anonymity of the data, the voluntary nature of the responses and the absence of any compensatory economic participation, as well as the time to complete the questionnaires, i.e. about $15 \mathrm{~min}$. The participants were also informed that they should answer only once. The informed consent was also made available and the participants could only advance in the completion of the instruments after indicating their agreement to participate in the study, a mandatory completion item. The questionnaires were available online between October and December 2020 and the process was completed by February 2020. The study was conducted in accordance with the Declaration of Helsinki and the protocol was approved and financed by the Portuguese Foundation for Science and Technology project "VAWDV in Times of Pandemic, namely, characterization, challenges and opportunities in RS," in July 2019.

\section{Variables and measures}

Background questionnaire. It included a series of questions about age, sex, academic qualification, scientific training area, training as victim support technicians and length of professional experience.

$R S$. Participants were requested to identify the type of support provided to VAWDV victims during the lockdown and the state of emergency resulting from the pandemic generated by COVID-19, as well as the type of support available before this pandemic situation. Participants were asked about the frequency with which they used the following types of support: telephone, email, social or mobile applications (e.g. WhatsApp), videoconference (e.g. Skype), through a five-point Likert-type scale ranging from 1 - never to 5 - always. In a second stage, the participants were asked about their training to provide RS; classifying their ability to use the types of support mentioned through a four-point Likert-type scale ranging from 1 - bad to 4 - very well); identifying, from a list presented the advantages (e.g. aid to deal with isolation; integration in self-help groups; promotion of victims' empowerment) and barriers (e.g. victim's reduced knowledge on digital technologies use; technical difficulties, such as weak internet signal) in the use of RS and the usefulness of RS and, furthermore; qualifying the professional experience in RS to victims of VAWDV, based on a four-point Likert-type scale ranging from 1 - bad to 4 - very good. Finally, and also using a five-point Likert-type scale ranging from 1 - very receptive to 5 - non-receptive, support professionals rated institutional policies regarding RS, classifying the frequency in the implementation of the different types of support (e.g. f2f, telephone, email, social applications and video conferente), including during the lockdown and state of emergency.

\section{Data analysis}

For descriptive statistical analysis, the Statistical Program for Social Sciences was used (IBM SPSS for Windows, version 27.0, IBM Corp, Armonk, NY, USA). The descriptive univariate analyzes were computed to characterize the sample, to identify the type of support most used by professionals (objective i), to characterize the type of support most provided in the lockdown (objective ii), to identify the RS modalities used during the state of emergency 
(objective iii), to characterize the training of professional support for RS victims, as well as training needs in this area (objective iv) and to know the institutional policies to implement RS (objective $\mathrm{v}$ ). The mode (Mo) was calculated as a measure of central tendency to better identify the value of the variable that appears most frequently (Field, 2017).

\section{Results}

\section{General support provided by professionals during the COVID-19 pandemic crisis lockdown}

During the lockdown, professionals have provided support to the victims through telephone more frequently ( $M o=$ always) than the remaining forms of support. Regarding the emergency state declared between March 19 and May 3 (2020), 82.1\% of the professionals reported that they continued to provide support to victims. Telephone support remained to be the most frequent support during this period (Mo = always) (Table 1).

When referring to the RS typically used, i.e. not strictly restricted to the COVID-19 pandemic period, support professionals referred using mostly the telephone.

\section{Training and needs of support professionals in remote support}

The majority of the support professionals stated that they did not have any professional qualification regarding RS with VAWDV ( $n=163,82.7 \%)$. Nonetheless, more than half have

\section{Table 1 Professional support during the lockdown and the emergency state}

\begin{tabular}{|c|c|c|c|c|}
\hline Support & Lockdown frequency & $N(\%)$ & Emergency state frequency & $N(\%)$ \\
\hline \multirow[t]{5}{*}{ Telephone } & Always & $86(43.9)$ & Always & $113(57.7)$ \\
\hline & Frequently & $73(37.2)$ & Frequently & $32(16.3)$ \\
\hline & Sometimes & $23(11.7)$ & Sometimes & $10(5.1)$ \\
\hline & Seldom & $6(3.1)$ & Seldom & $8(4.1)$ \\
\hline & $\begin{array}{l}\text { Never } \\
\text { (Mo = Always) }\end{array}$ & $8(4.1)$ & $\begin{array}{l}\text { Never } \\
\text { (Mo = Always) }\end{array}$ & $33(16.8)$ \\
\hline \multirow[t]{5}{*}{ E-mail } & Always & $25(12.8)$ & Always & $36(18.4)$ \\
\hline & Frequently & $51(26)$ & Frequently & $42(21.4)$ \\
\hline & Sometimes & $48(24.5)$ & Sometimes & $34(17.3)$ \\
\hline & Seldom & $27(13.8)$ & Seldom & $25(12.8)$ \\
\hline & $\begin{array}{l}\text { Never } \\
\text { (Mo = Frequently) }\end{array}$ & $45(23)$ & $\begin{array}{l}\text { Never } \\
\text { (Mo = Never) }\end{array}$ & $59(30.1)$ \\
\hline \multirow{5}{*}{$\begin{array}{l}\text { Social applications } \\
\text { (e.g. WhatsApp) }\end{array}$} & Always & $9(4.6)$ & Always & $14(7.1)$ \\
\hline & Frequently & $32(16.3)$ & Frequently & $26(13.3)$ \\
\hline & Sometimes & $35(17.9)$ & Sometimes & $29(14.8)$ \\
\hline & Seldom & $37(18.9)$ & Seldom & $25(12.8)$ \\
\hline & $\begin{array}{l}\text { Never } \\
\text { (Mo = Never) }\end{array}$ & $82(41.8)$ & $\begin{array}{l}\text { Never } \\
\text { (Mo = Never) }\end{array}$ & $102(41.8)$ \\
\hline Videoconference (e.g. & Always & $8(4.1)$ & Always & $14(7.1)$ \\
\hline \multirow[t]{4}{*}{ Skype) } & Frequently & $36(18.4)$ & Frequently & 28 (14.3) \\
\hline & Sometimes & $25(12.8)$ & Sometimes & $22(11.2)$ \\
\hline & Seldom & $36(18.4)$ & Seldom & $18(9.2)$ \\
\hline & $\begin{array}{l}\text { Never } \\
\text { (Mo = Never) }\end{array}$ & $91(46.4)$ & $\begin{array}{l}\text { Never } \\
\text { (Mo = Never) }\end{array}$ & $114(58.2)$ \\
\hline \multirow[t]{5}{*}{ Presencial } & Always & $18(9.2)$ & - & - \\
\hline & Frequently & $53(27)$ & - & - \\
\hline & Sometimes & $66(33.7)$ & - & - \\
\hline & Seldom & $36(18.4)$ & - & - \\
\hline & $\begin{array}{l}\text { Never } \\
\text { (Mo = Sometimes) }\end{array}$ & $23(11.7)$ & - & - \\
\hline
\end{tabular}


expressed to consider RS a good ( $n=97,49.5 \%)$ and a very useful $(n=124,63.3 \%)$ tool for helping VAWDV victims.

Concerning the RS that support professionals consider more suitable for assisting the VAWDV victims, most mention the telephone $(n=174,39.4 \%)$ and videoconference support $(n=162,36.6 \%)$.

They also reported feeling generally prepared to provide any kind of RS (telephone: Mo = very well; email: $M o=$ very well; social applications: $M o=$ well; videoconference: $M o=$ well) (Table 2).

\section{Remote support provided by support professionals: advantages, obstacles, experience and utility}

The support professional underlined several advantages of RS. The most frequently reported advantage was decreasing victim isolation ( $n=172 ; 21.6 \%)$. However, when considering obstacles that this type of RS may present, professionals most frequently mentioned the victims' difficulty in accessing and using digital technologies ( $n=171$, $29.9 \%)$ and the victims' reduced knowledge on digital technologies use ( $n=162,28.5 \%$ ). Nonetheless, support professionals declared to have a good experience using RS ( $n=97$, $49.5 \%)$ and consider it to be a very useful tool ( $n=124,63.3 \%)$ to support VAWDV (Table 3). Considering the risks involved in RS, support professionals do not think there is any risk of RS to professionals ( $n=125,63.8 \%)$. However, professionals believe there are risks for women seeking RS $(n=143,73.0 \%)$. Some of the risks identified by the support professionals were as follows: control of digital devices by the aggressor (45\%) (e.g. telephone contacts, messages or emails may be intercepted by the alleged aggressor); the existence of cohabitation and/or offender-victim proximity (39\%) (e.g. Risks in case of cohabitation with the aggressor); possible retaliation against the victim and increased risk of revictimization (38\%) (e.g. The aggressor listens to the victim's report and confronts him,

\section{Table 2 RS level of preparation by support professionals}

\begin{tabular}{llc} 
Support & Preparation & $N(\%)$ \\
\hline Telephone & Very well & $136(69.4)$ \\
& Well & $50(25.5)$ \\
& Reasonable & $9(4.6)$ \\
& Bad & $0(0)$ \\
& Very bad & $1(0.5)$ \\
& (Mo = very well) & \\
Email & Very well & $111(56.6)$ \\
& Well & $54(27.6)$ \\
& Reasonable & $24(12.2)$ \\
& Bad & $7(3.6)$ \\
Social/mobile applications & Very bad & $0(0)$ \\
(e.g. WhatsApp) & (Mo = very well) & \\
& Very well & $72(36.7)$ \\
& Well & $78(39.8)$ \\
& Reasonable & $35(17.9)$ \\
Videoconference (e.g. & Bad & $8(4.1)$ \\
Skype) & Very bad & $3(1.5)$ \\
& (Mo = well) & $64(32.7)$ \\
& Very well & $86(43.9)$ \\
& Well & $38(19.4)$ \\
Note: $N=$ Number of cases & Reasonable & $6(3.1)$ \\
& Bad & $2(1.0)$ \\
& Very bad &
\end{tabular}




\begin{tabular}{llc}
\hline Advantages & Aid to deal with isolation & $172(21.6)$ \\
& Reduce inhibition, fear and shame & $143(18.0)$ \\
& Reduce financial costs & $143(18.0)$ \\
& Promotion of victims' empowerment & $120(15.1)$ \\
& Access to risk management devices and safety planning & $104(13.0)$ \\
& Integration in self-help groups & $36(4.5)$ \\
& Victim's difficulty in accessing and using digital technologies & $171(29.9)$ \\
& Victim's reduced knowledge on digital technologies used & $163(28.5)$ \\
Obstacles & Technical difficulties (e.g. weak internet signal) & $142(24.9)$ \\
& Victim's difficulty in the selection of the most suitable digital technology by victims \\
& Very good & $95(16.7)$ \\
Experience & Good & $26(13.3)$ \\
& Reasonable & $97(49.5)$ \\
& Bad & $45(23.0)$ \\
& Very bad & $4(2.0)$ \\
Utility & Very useful & $0(0)$ \\
& Useful & $124(63.3)$ \\
& Reasonably useful & $56(28.6)$ \\
& Barely useful & $15(7.8)$ \\
Note: $N=$ Number of cases & & $1(0.5)$ \\
& & $0(0)$
\end{tabular}

which can lead to more serious situations of aggression), confidentiality issues (32\%) (e.g. Confidentiality management).

\section{Institutional policies to implement remote support}

During the emergency state period in the COVID-19 pandemic, more than half of support professionals worked solely remotely, i.e. teleworking $(n=103,60.2 \%)$. Considering the remaining support interventions, $25.2 \%$ worked both f2f and remotely $(n=43)$ while $14.6 \%$ only worked in f2f $(n=25)$. In more than half of the cases, RS was promoted in the institutions where professionals work ( $n=102,52.0 \%)$, actively supported the use of RS (telephone, email, social applications, videoconference: $M o=$ very receptive) (Table 4).

Concerning how the support institutions have adapted to the COVID-19 pandemic, 57.7\% have suspended the f2f support $(n=113), 23.5 \%$ continued their normal functioning $(n=$ 46), $18.4 \%$ adapted other measures $(n=36)$ and only one institution $(0.5 \%)$ closed their services. After the lockdown, $48.0 \%$ of the institutions resumed their normal functioning ( $n=$ 94), $44.9 \%$ adopted mix support, i.e. f2f and RS ( $n=88), 6.1 \%$ adopted other strategies $(n=12)$ and $1.0 \%$ suspended f2f support $(n=2)$.

\section{DISCUSSION}

The outbreak of the COVID-19 pandemic brought multiple challenges for the support provided to VAWDV victims, as it was necessary to guarantee the continuity of support while maintaining compliance with the public health security measures imposed by the situation experienced (e.g. restrictions on mobility, the need for masks and social distancing, self-isolation rules and the closing of non-essential services). This created the need for a reorganization of services and additional effort by support professionals to better respond to these demands and the pressure they represent. In the present study, it was intended to analyze the type of support provided by professionals working with victims of VAWDV during the lockdown period and the state of emergency in the COVID-19 pandemic in Portugal, as well as to understand their level of training, to know the different specificities underlying RS and to know the institutional policies adopted. 
Table 4 Receptivity of support professionals institutions regarding RS

\begin{tabular}{llr} 
Support & Receptivity & $N(\%)$ \\
\hline Telephone & Very receptive & $151(77.0)$ \\
& Receptive & $33(16.8)$ \\
& Reasonably receptive & $8(4.1)$ \\
& Barely receptive & $4(2.0)$ \\
& Not receptive & $0(0)$ \\
Email & (Mo = very receptive) & \\
& Very receptive & $137(69.9)$ \\
& Receptive & $43(21.9)$ \\
& Reasonably receptive & $10(5.1)$ \\
Social/Mobile Applications & Barely receptive & $5(2.6)$ \\
(e.g. WhatsApp) & Not receptive & $1(0.5)$ \\
& (Mo = very receptive) & $105(53.6)$ \\
& Very receptive & $46(23.5)$ \\
& Receptive & $23(11.7)$ \\
Videoconference (e.g. & Reasonably receptive & $16(8.2)$ \\
Skype) & Barely receptive & $6(3.1)$ \\
& Not receptive & $102(52.0)$ \\
& (Mo = very receptive) & $51(26.0)$ \\
Note: $N=$ Number of cases & Very receptive & $26(13.3)$ \\
& Receptive & $12(6.1)$ \\
& Reasonably receptive & $5(2.6)$
\end{tabular}

The telephone emerges as the frequently used form of support to victims of VAWDV during the lockdown (43.9\%), emergency state $(57.1 \%)$ and in general $(50.5 \%)$, confirming hypothesis 1 . In fact, telephone assistance is a very used service that has been delivered in several countries for a relatively long period (Reese et al., 2006). During the lockdown, a considerable percentage of support professionals (33.7\%) also reported maintaining f2f help or use email (26.1\%). Indeed, the literature shows that it is feasible to use different forms of RS, e.g. telephone, email and chat, to provide help (Haberstroh et al., 2008), also defending a mixing online and f2f approach (Wentzel et al., 2016). Furthermore, RS using various digital solutions has been identified as an important and necessary alternative in supporting victims in crisis situations, such as that generated by COVID-19 (Anka et al., 2020). It has also been argued that a combination of online and f2f support, i.e. blended support, should be used to their fullest potential to create an ideal combination of both types of support. They should respect some guidelines such as online and offline modalities must be interconnected in some way and not constituting separate support pathways; blended support must be dynamic and flexible, presenting the content in a non-linear and dynamic way through texts, images or interactive assignments; the type of online support must be carefully selected and adjusted to the person's specific situation, needs and abilities (e.g. characteristics and skills of victims, severity and type of violence); and, finally, support professionals should analyze and discuss with victims the rationale for using blended support, to ensure the appropriate connection between technology and victims (Wentzel et al., 2016). In addition, existing evidence demonstrates that online support is considered equivalent to f2f help (Murphy et al., 2009) and to support services provided by telephone (Fukkink and Hermanns, 2009b). Others (Fukkink and Hermanns, 2009a) argue that online support can be considered better than telephone support. Nevertheless, the present study reveals some resistance from professionals to seek online support. Considering the mode in relation to the use of other digital solutions to provide support, a significant percentage of participants reported to have never used any social/mobile applications (e.g. WhatsApp) (41.8\% vs $41.8 \%$ ) or videoconference (e.g. Skype) (46.4\% vs 
$58.2 \%)$, in both periods assessed, i.e. lockdown and emergency state, respectively. These results may be explained by the fact that most participants (82.7\%) assume that they have no training with online support to victims of VAWDV, as expected in H2. Furthermore, the greater use of the telephone, opposite to other forms of RS, may also be due to the fact that technological/digital innovations have not previously been considered strategically. In this sense, systems and training have not been put in place to support professionals to use digital technologies in supporting VAWDV victims. Therefore, it is necessary as follows: the existence of equipment available for this purpose, such as smartphones, the existence of secure ICT systems, especially if professionals are working from home, the existence of protocols on the use of videoconferences (e.g. always blurring the background to prevent identification) or even addressing the use of the email address (e.g. email that the offender can see) and, the lack of experience, training and support to use a wider range of tools for remote working, for example, on safety planning of RS with VAWDV victims.

Nevertheless, and in general, the support professionals considered themselves prepared to provide any type of RS (telephone: $M O=$ very well; email: $M O=$ very well; social applications: $M O=$ well; videoconference: $M o=$ well). More than half of the support professionals have qualified RS as good (49.5\%) and a very useful type of support (63.3\%).

Digital solutions, such as websites, email and/or social networks, are gradually emerging as valid methods for addressing VAWDV remotely or to support victims in general (Caridade and Dinis, 2020). The professionals inquired in the present study recognized several advantages in using RS such as:

- To deal with isolation (21.6\%);

- To reduce inhibition, fear and shame (18\%);

- To reduce financial cost (18.0\%); and

- To promote the victims' empowerment (15.1\%).

The literature has been identifying the numerous opportunities encouraging the use of digital platforms, particularly in terms of disseminating essential victim support resources and services, enabling the creation of online support groups to reduce the feeling of isolation and empowering the victims (Al-Alosi, 2020). In fact, and often, victims of VAWDV find themselves in a situation of great isolation, due to the victimization process by their partners and with reduced access to social support resources (Capaldi et al., 2012), so digital solutions are an important resource for obtaining information or even sharing experiences of violence and seeking help (Anderson et al., 2019). Also, Constantino et al. (2014) reported that online environments make it possible to better deal with social risk and inhibition, reducing them and promoting the sharing of unwanted thoughts and feelings by the victims.

The support professionals also identify some obstacles and risks associated to the use of RS. More specifically, the victims' difficulty in accessing and using digital technologies (29.9\%) and the victims' reduced knowledge on digital use of technologies (28.5\%) were the most frequent obstacles reported by the support professionals, corroborating what is reported in other works (Al-Alosi, 2020; Wentzel et al., 2016). Although support professionals do not recognize any risk of RS for themselves (63.8\%), they contemplated the existence of risk in RS for victims (73.0\%). Concern about the safety of victims of VAWDV has been effectively discussed, either because offenders can remotely monitor their devices, such as computers, phones and home security systems or because of the aggressors' location of victims, putting their physical safety at risk (Finn and Atkinson, 2009). The existence of the potential risks and obstacles associated with the use of ICT and RS, should not, however, lead to avoiding and disregarding the potential of digital solutions in supporting the victims of VAWDV. To find control strategies or mechanisms to the most diverse challenges that the use of ICT imposes, should be the way forward (AI-Alosi, 2020; 
Caridade and Dinis, 2020). In addition, strategies have been pointed out to promote the safety of victims when using the digital platforms, for instance: using applications that increase the protection of the victims, such as password protected sites, "quick exit" buttons on sites, particularly relevant when the victim still lives with the abuser (Sorenson et al., 2014) or promoting e-literacy skills to minimize the risk (e.g. teaching about online privacy settings, providing information about password protection, locking and filtering the software used in online contact) (Rempel et al., 2018).

Positive results were found in what concerns the institutional policies to implement RS. Thus, in more than half of the cases, RS was promoted in the institutions where the support professionals work (52.0\%), being very receptive overall (telephone, email, social/mobile applications, videoconference: $M O=$ very receptive). During the COVID-19 pandemic situation, $57.7 \%$ of the service providers suspended f2f support, $23.5 \%$ continued their normal functioning and $18.4 \%$ have adapted other measures. After the lockdown, $48.0 \%$ of the institutions returned their normal functioning, $44.9 \%$ adopted mix support, i.e. f2f and RS and $6.1 \%$ adopted other types of functioning. Indeed, the importance of f2f victim support services has been documented, considering that the use of digital solutions should constitute a complementary strategy to respond to VAWDV (Chayn and SafeLives, 2017) and to be implemented in situations of crisis, such as the one generated by the COVID-19 pandemic situation (EIGE, 2021). It is, therefore, necessary to develop financing policies that can increase the type of response given by support services to also include online tools (Chayn and SafeLives, 2017). It can then be concluded that $H 3$ has been partially confirmed. Thus, and if, on the one hand, there was some opening of institutions for the use of RS, on the other hand, the use of digital solutions seems restricted by the scarcity of resources and, above all, with respect to the training and preparation of support professionals involved in RS.

This study has some limitations that must be considered when assessing the results. This is an exploratory study, conducted only with a sample $(n=196)$ of support professionals who are part of the NSNVDV, within a universe of $N=300$ registered professionals. Therefore, other institutions that support victims (e.g. academic institutions that provide support services to victims of VAWDV) who are not part of this support network were not considered, something that should be attended in future studies. Given the exploratory nature of this study, only descriptive analyzes were conducted. It is also important to develop other in-depth studies that seek to analyze, potentialities, obstacles and risks perceived in the use of the RS, involving the victims of VAWDV. Because certain groups, such as individuals who identify as lesbian, gay, bisexual and/or transgendered, are often neglected or overlooked as potential survivors of interpersonal violence (Carballea and Rivera, 2020), they should also be included in future studies. Considering the proven potential of blended care in terms of mental health (Wentzel et al., 2016), as well as the documented usefulness of RS in crisis situations, such as COVID-19 (Anka et al., 2020), it is important to continue to promote research to reach the full potential of blended and RS, trying to understand what suits who and how technology features and digital solutions can be optimized to assist the victims of DV. Research in this area should also progress toward randomized studies that allow the comparison of online intervention with f2f efforts in assisting the VAWDV victims. It is also important to give the voice to the victim in assessing the effectiveness of RS. Indeed, the reconciliation of factors commonly present in victims, e.g. isolation, difficulty in seeking help via f2f for different reasons that may include social stigma, guilt, humiliation or shame, with the advantages inherent in the use of ICT, makes this an important area of future research. It is important to continue investing in the development of new types of online support to prevent DV and support victims, always considering the process of the reliability of the assessment tools used or the impact on reducing the future risk of DV (Caridade and Dinis, 2020). Finally, it has been argued the creation and availability of different digital tools (e.g. infographics, factsheets, podcasts, webinars, videos and articles centered on psychoeducation and evidence-based 
interventions), which are able to consume and see at home, should focus both victims and aggressors, promoting emotional regulation skills and communication strategies to better manage the stressors caused by the COVID-19 pandemic (Carballea and Rivera, 2020) or other situations of crisis.

\section{Conclusions}

The present study aims to bring an important and innovative contribution in terms of research on the type of support provided to VAWDV by the support professionals in the Portuguese context during the COVID-19 pandemic. Other studies focusing on this specific context are unknown when considering the Portuguese reality. The results of this study prove that the telephone constituted the type of support most used during the period of the first Portuguese lockdown and the state of emergency (March to May 2020), also concluding by the reduced use of other digital solutions by the support professionals assisting the VAWDV victims. Although the support professionals consider themselves capable of using other types of RS, they recognize that they do not have the appropriate knowledge and training to do it. Additionally, and if, on the one hand, the professionals recognize the multiple benefits of the RS, they also admitted the risks that the RS may represent, particularly to the victims. Victim support services proved to be very receptive and available to implement other support modalities for victims of VAWDV.

The findings from this study have important implications for promoting the performance of support professionals in assisting the victims of VAWDV and in improving the service providers in crisis situations, such as the one generated by the COVID-19 pandemic. First, it is essential to provide education and training to support professionals who assist the victims of VAWDV in the use and handling of the most diverse digital solutions (e.g. social applications such as WhatsApp, videoconference by Skype, teams, Zoom, Google Meet) and other available digital products (e.g. infographics, factsheets, podcasts, webinars, videos) that constitute fundamental tools to enhance the support to VAWDV victims. Furthermore, it is also important to make financial resources available so that the support institutions can provide the necessary resources to offer additional support mechanisms to victims of VAWDV.

\section{References}

Al-Alosi, H. (2020), "Fighting fire with fire: exploring the potential of technology to help victims combat intimate partner violence", Aggression and Violent Behavior, Vol. 52, pp. 101-356.

Anderson, E., McClelland, J., Krause, C.M. ... (2019), and Koss. M. "Web-based and mHealth interventions for intimate partner violence prevention: a systematic review", Trauma, Violence \& Abuse, pp. 1-15.

Anka, A., Thacker, H. and Penhale, B. (2020), "Safeguarding adults practice and remote working in the COVID-19 era: challenges and opportunities", The Journal of Adult Protection, Vol. 22 No. 6, pp. 391-399.

Bocioaga, A. (2020), ESSS Outline: Service Response to Domestic Abuse during COVID-19, IRISS, Glasgow.

Boserup, B., McKenney, M. and Elkbuli, A. (2020), "Alarming trends in US domestic violence during the COVID-19 pandemic", The American Journal of Emergency Medicine, Vol. 38 No. 12, pp. 2753-2755.

Boxall, H., Morgan, A. and Brown, R. (2020), The prevalence of domestic violence among women during the COVID-19 pandemic", Australian Institute of Criminology: Statistical Bulletin, available at: www.aic. gov.au/sites/default/files/2020-07/sb28_prevalence_of_domestic_violence_among_women_during_Covid19_pandemic._Pdf (accessed 23 march 2021).

Bradbury-Jones, C. and Isham, L. (2020), "The pandemic paradox: the consequences of COVID-19 on domestic violence", Journal of Clinical Nursing, Vol. 29 No. 13-14, pp. 2047-2049.

Capaldi, D., Knoble, N., Shortt, J. and Kim, H. (2012), "A systematic review of risk factors for intimate partner violence", Partner Abuse, Vol. 3 No. 2, pp. 231-280. 
Carballea, D. and Rivera, R. (2020), "Coronavirus and interpersonal violence: a need for digital mental health resources", Psychological Trauma: Theory, Research, Practice, and Policy, Vol. 12 No. S1, pp. S208-S209.

Caridade, S. and Dinis, M.A. (2020), "Distance learning and social issues: opportunities and challenges in preventing violence", in Moura, A., Reis, P. and Cordeiro, N. (Eds), Handbook of Research on Determining the Reliability of Online Assessment and Distance Learning, IGI Global, pp. 443-460.

Center for Disease Control and Prevention (2016), "Understanding teen dating violence. Fact sheet. 2016", available at: www.cdc.gov/violenceprevention/pdf/teen-dating-violencefactsheet-a. pdf (accessed 16 January 2021).

Commission for Citizenship and Gender Equality (2020), App VD - APPoio Contra a Violência Doméstica [App VD - Support Against Domestic Violence], available at: www.cig.gov.pt/area-portalda-violencia/violencia-contra-as-mulheres-e-violencia-domestica/campanhas/app-vd-appoio-contra-aviolencia-domestica-2018/(accessed 10 August 2021).

Constantino, R., Hamdan-Mansour, A.M., Henderson, A., Noll-Nelson, B., Doswell, W. and Braxter, B. (2014), "Assessing the readability and usability of online H-E-L-P intervention for IPV survivors", Open Journal of Nursing, Vol. 4 No. 3.

European Institute for Gender Equality (EIGE) (2021), "The covid-19 pandemic and intimate partner violence against women in the EU", available at: https://eige.europa.eu/publications/covid-19-pandemicand-intimate-partner-violence-against-women-eu?fbclid=IwAR26IOBvH67wLQZtNLfraBTYgEOmb16bnmEA w8I884LI3_eoubFf9juSC-s (accessed 23 March 2021).

Faias, J., Caridade, S. and Cardoso, J. (2016), "Exposição à violência familiar e abuso íntimo em jovens: que relação?[ exposure to family violence and intimate abuse in young people: what relationship?]", Psychologica, Vol. 59 No. 1, pp. 7-23.

Field, A. (2017), Discovering Statistics Using IBM SPSS Statistic, 2nd ed., Sage, New York, NY.

Finn, J. and Atkinson, T. (2009), "Promoting the safe and strategic use of technology for victims of intimate partner violence: evaluation of the technology safety project”, Violence against Women, Vol. 15 No. 11, pp. 1402-1414.

Ford-Gilboe, M., Varcoe, C., Scott-Storey, K., Wuest, J., Case, J., Currie and Wathen, N. (2017), "A tailored online safety and health intervention for women experiencing intimate partner violence: the iCAN plan 4 safety randomized controlled trial protocol", BMC Public Health, Vol. 17 No. 1, p. 273.

FRA (2014), "Violence against women: an EU-wide survey", FRA - European Union Agency for Fundamental Rights.

Fukkink, R. and Hermanns, J. (2009a), "Counseling children at a helpline: chatting or calling?", Journal of Community Psychology, Vol. 50 No. 6, pp. 759-766.

Fukkink, R. and Hermanns, J. (2009b), "Children's experiences with chat support and telephone support", Journal of Child Psychology and Psychiatry, Vol. 50 No. 6, pp. 759-766.

Glass, N., Clough, A., Case, J., Hanson, J., Barnes-Hoyt, J., Waterbury, A., ... Perrin, N. (2015), "A safety app to respond to dating violence for college women and their friends: the MyPlan study randomized controlled trial protocol", BMC Public Health, Vol. 15 No. 1, pp. 871-884.

Haberstroh, S., Parr, G., Bradley, L. and Gee, R. (2008), "Facilitating online counseling: perspectives from counselors in training", Journal of Counseling \& Development, Vol. 86 No. 4, pp. 460-470.

Hegarty, K., Tarzia, L., Valpied, J., Murray, E., Humphreys, C., Taft, A., ... Glass, N. (2019), "An online healthy relationship tool and safety decision aid for women experiencing intimate partner violence (IDECIDE): a randomised controlled trial", The Lancet Public Health, Vol. 4 No. 6, pp. e301-e310.

Murray, C.E., Chow, A.S., Pow, A.M., Croxton, R. and Poteat, L. (2015), "Domestic violence service providers' technology readiness and informationnNeeds", Journal of Aggression, Maltreatment \& Trauma, Vol. 24 No. 3, pp. 257-278, doi: 10.1080/10926771.2015.997908.

Neves, S. and Nogueira, C. (2010), "Deconstructing gendered discourses of love, power and violence in intimate relationships", in Dana J. and Alisha, A. (Eds), Silencing the Self across Cultures Depression and Gender in the Social World, University Press, Oxford, pp. 241-261.

Neves, S., Cameira, M., Machado, M., Duarte, V. and Machado, F. (2016), "Beliefs on marital violence and self-reported dating violence: a comparative study of cape verdean and portuguese adolescents", Journal of Child and Adolescent Trauma. 
Paulino, M., Dumas-Diniz, R., Brissos, S., Brites, R., Alho, L., Simões, M.R. and Silva, C.F. (2021), "COVID-19 in Portugal: exploring the immediate psychological impact on the general population", Psychology, Health \& Medicine, Vol. 26 No. 1, pp. 44-55.

Reese, R., Conoley, C. and Brossart, D. (2006), "The attractiveness of telephone counseling: an empirical investigation of client perceptions”, Journal of Counseling \& Development, Vol. 84 No. 1, pp. 54-60.

Rempel, E., Lorie, D., Jodi, H. and Rodger, S. (2018), "Intimate partner violence: a review of online interventions", Informatics for Health and Social Care, Vol. 44 No. 2, pp. 204-219.

Runyan, C., Gunther-Mohr, C., Orton, S., Umble, K., Martin, S. and Coyne-Beasley, T. (2005), "PREVENT. A program of the national training initiative on injury and violence prevention", American Journal of Preventive Medicine, Vol. 29 No. 5, pp. 252-258.

Sabri, B., Njie-Carr, V., Messing, J., Glass, N., Brockie, T., Hanson, G., Case, J. and Campbell, J. (2019), "The weWomen and our-Circle randomized controlled trial protocol: a web-based intervention for immigrant, refugee and indigenous women with intimate partner violence experiences", Contemporary Clinical Trials, Vol. 76, pp. 79-84.

Safe Lives (2020), "Domestic abuse frontline service COVID-19 survey results", available at: https:// safelives.org.uk/sites/default/files/resources/SafeLives\%20survey\%20of\%20frontline\%20domestic\% _20abuse\%20organisations\%20for\%20COVID19\%2030.03.20_0.pdf (accessed 2 march 2021).

Sorenson, S., Shi, R., Zhang, J. and Xue, J. (2014), "Self-Presentation on the web: agencies serving abused and assaulted women", American Journal of Public Health, Vol. 104 No. 4, pp. 702-707.

SSI - Sistema de Segurança Interna (2021), "Relatório anual de segurança interna 2020 [domestic violence 2020 - annual monitoring report]”, Sistema de Segurança Interna, Lisboa.

Trabold, N., McMahon, J., Alsobrooks, S., Whitney, S. and Mittal, M. (2018), "A systematic review of intimate partner violence interventions: state of the field and implications for practitioners", Trauma, Violence \& Abuse, Vol. 21 No. 2, pp. 311-325.

United Nations (1993). "Declaration on the elimination of violence against women proclaimed by general assembly resolution 48/104 of 20 December 1993", available at https://www.un.org/en/genocideprevention/ documents/atrocity-crimes/Doc.21_declaration\%20elimination\%20vaw.pdf (accessed 23 March 2021).

Usher, K., Bhullar, N., Durkin, J., Gyamfi, N. and Jackson, D. (2020), "Family violence and COVID-19: increased vulnerability and reduced options for support", International Journal of Mental Health Nursing, Vol. 29 No. 4, pp. 549-552.

Walklate, S., Godfrey, B. and Richardson, J. (2021), "Changes and continuities in police responses to domestic abuse in England and Wales during the covid-19 'lockdown", Policing and Society, doi: 10.1080/10439463.2021.1896514.

Wentzel, J., van der Vaart, R., Ernst, B. and van Gemert-Pijnen, J. (2016), "Mixing online and face-to-face therapy: how to benefit from blended care in mental health care", JMIR Mental Health, Vol. 3 No. 1, p. 9.

World Health Organization (2013), "Global and regional estimates of violence against women: prevalence and health effects of intimate partner violence and non-partner sexual violence", World Health Organization, Geneva.

Yenilmez, M.I. (2020), "The covid-19 pandemic and the struggle to tackle gender-based violence", The Journal of Adult Protection, Vol. 22 No. 6, pp. 391-399.

\section{Further reading}

Chayn, S. and Lives, S. (2017), "Tech vs. Abuse: research findings. Comic relief", available at: www. techvsabuse.info/research-findings (accessed 23 March 2021).

\section{Author affiliations}

Sónia Maria Martins Caridade is based at the Faculty of Human and Social Sciences, University Fernando Pessoa, Porto, Portugal, and Interdisciplinary Center for Gender Studies (CIEG) of the Higher Institute of Social and Political Sciences, University of Lisbon, Lisbon, Portugal.

Rosa Saavedra is based at the Portuguese Association for Victim Support (APAV), Lisbon, Portugal and Faculty of Law, School of Criminology, Interdisciplinary Research Centre on Crime, Justice and Security (CJS), University of Porto, Porto, Portugal. 
Rita Ribeiro is based at the Faculty of Psychology and Educational Sciences, Research Unit of the Cognitive-Behavioral Research and Intervention Center, University of Coimbra, Coimbra, Portugal, and Laboratory of Psychology (LabPSI), Egas Moniz Higher Institute of Health Science, Multidisciplinary Research Center of Egas Moniz (CiiEM), Almada, Portugal.

Ana Cristina Oliveira and Manuela Santos are both based at the Portuguese Association for Victim Support (APAV), Lisbon, Portugal.

Iris Sofia Almeida is based at the Department of Laboratory of Psychology (LabPSI), Egas Moniz Higher Institute of Health Science, Multidisciplinary Research Center of Egas Moniz (CiiEM), Almada, Portugal.

Cristina Soeiro is based at the Portuguese Association for Victim Support (APAV), Lisbon, Portugal and Laboratory of Psychology (LabPSI), Egas Moniz Higher Institute of Health Science, Multidisciplinary Research Center of Egas Moniz (CiiEM), Almada, Portugal.

\section{Corresponding author}

Sónia Maria Martins Caridade can be contacted at: soniac@ufp.edu.pt

For instructions on how to order reprints of this article, please visit our website: www.emeraldgrouppublishing.com/licensing/reprints.htm

Or contact us for further details: permissions@emeraldinsight.com 